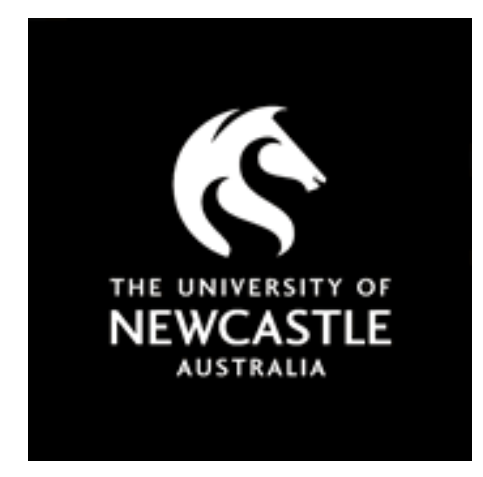

NOVA

University of Newcastle Research Online

nova.newcastle.edu.au

Khan, Uzair ljaz, Chen, Zhiyong. "Natural oscillation in compass-gait biped robots.", Published in Proceedings of the 2016 Australian Control Conference (AuCC). (2016)

Available from: http://dx.doi.org/10.1109/AUCC.2016.7867922

(C) 2016 IEEE. Personal use of this material is permitted. Permission from IEEE must be obtained for all other uses, in any current or future media, including reprinting/republishing this material for advertising or promotional purposes, creating new collective works, for resale or redistribution to servers or lists, or reuse of any copyrighted component of this work in other works.

Accessed from: http://hdl.handle.net/1959.13/1347526 


\title{
Natural Oscillation in Compass-gait Biped Robots
}

\author{
Uzair Ijaz Khan and Zhiyong Chen
}

\begin{abstract}
The compass-gait biped model is a unique, yet simple model, often used as a test bench for new control strategies in the field of legged locomotion. This paper presents a novel continuous mathematical model for compass-gait biped robots, modeling the discontinuities in impact with a nonlinear function. Natural oscillation is defined as a free response under the damping compensation to achieve persistent oscillation. The phenomenon of natural oscillation was previously exploited in the linear dynamics of multi-segmental locomotor, and it is now extended to this humanoid system with the contact force from the ground modelled with a nonlinear bump function.
\end{abstract}

\section{INTRODUCTION}

Animal locomotion can be very complex to analyze especially with their complicated neuromuscular and skeletal systems, and the coordination between these systems. Simplified models with links representing limbs and muscles were presented in [1], [2] and [3]. To analyze human walking models, passive biped walkers were presented in [4], [5]. Passive walkers are of profound interest as they can walk on their own on a slope without any external energy source. The earliest works on dynamic walkers were initiated by Tad McGeer [6], investigating the passive gait through mathematical modeling. Following his work, Goswami et al. [7], [8] produced a series of papers based on biped walkers utilizing the event based impact modeling. More recently, a succession of literature on walking and running of energy efficient planar passive bipeds was produced in [9], [10].

Lately, compass-gait biped has emerged as a test bench for control strategies in legged locomotion [11]. It derives its similarities with the model of double inverted pendulum and its derivation involves the frequently used Euler-Lagrange equation. Even though the kinematics of the compass-gait model are simple, its analysis is quite complicated because of the hybrid nature of the model. The problem with such modeling is the presence of discontinuous terms in the differential equation, which can cause inconvenience in its analysis. Hence, the focus is shifting to remedy this and continuous mathematical models are developed. In [12], one such continuous force-based contact model is presented while also pointing out that the use of impact modeling could lead to artificial gaits which are not a representation of reality, as they rely on the assumption that the heel strike impacts are instantaneous, plastic and no sliding occurs.

A new mathematical model is discussed in this paper whereby a nonlinear bump function caters for the discontinuities resulted by the impact. This model ensures that the dynamics are captured in one differential equation, hence eliminating the need for inconvenient hybrid model.

Once the mathematical model was developed, it was then checked for the presence of natural oscillations. It is believed that exploiting the phenomenon of resonance during locomotion can lead to energy efficient gaits [13]. Natural oscillation is defined as a free response to a modified system by removing a proper amount of damping effects to achieve persistent oscillation. It is shown in [14] that if the damping is compensated by a proper amount, the linear system can achieve marginal stability, i.e. the natural oscillations, which is analytically characterized in terms of a generalized eigenvalue of the mass-stiffness matrix pair. This definition was tested on various mathematical models of snake-like locomotors and other such models, and was thus verified by their results [15], [16]. The main objective of this paper to investigate the concept in a nonlinear compass-gait model.

\section{Mathematical Modeling}

The model consists of two links, each with distributed mass representing each leg, connected together by a hip joint, which represents the upper body as shown in Figure 1. The legs have springs attached beneath them to provide a force at the time of impact. The mathematical model is derived from the standard Euler-Lagrange equation.

Let $q=\left[q_{1}, q_{2}\right]^{T}$ be a vector of generalized coordinates in the configuration space as shown in Figure 1. The Lagrangian $\mathscr{L}$ is given by

$$
\mathscr{L}(q, \dot{q})=U(q, \dot{q})-V(q)
$$

where $U(q, \dot{q})$ represents the total kinetic energy of the system and $V(q)$ the potential energy of the system. Consider the Lagrangian formula,

$$
\frac{d}{d t} \frac{\partial \mathscr{L}}{\partial \dot{q}}-\frac{\partial \mathscr{L}}{\partial q}=B u,
$$

which yields the equation of motion

$$
M(q) \ddot{q}+C(q, \dot{q}) \dot{q}+D(q) \dot{q}+K_{o} q+G(q)=T_{c}(q, \dot{q}) .
$$

Here $M(q)$ is positive definite representing the moment of inertia matrix, $C(q, \dot{q})$ is Coriolis, $D(q)$ is the damping matrix, $K_{o}$ represents the joint stiffness, $G(q)$ denotes the gravitational torque, and $T_{c}(q, \dot{q})$ is the torque from the contact force of the ground. For simplicity, the Rayleigh damping $(D=\mu M)$ was considered.

The system is linearized to simplify the equation (2). After suitable damping compensation with $\varepsilon$ for persistent oscillation, the equation becomes

$$
M(q) \ddot{q}+(\mu-\varepsilon) M(q) \dot{q}+\left(K_{o}+G\right) q=T_{c}(q, \dot{q}) .
$$

The matrix $K_{o}$ in (3) is a symmetric matrix representing the body stiffness, and $T_{c}$ denotes the torques acting on the 


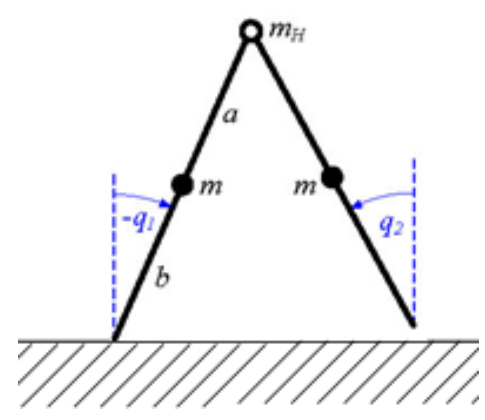

Fig. 1. Schematic diagram of a compass-like biped robot. [17]

system externally. The contact torque vector, $T_{c}$, consists of $T_{c_{1}}$ and $T_{c_{2}}$, one for each leg, and it is given by

$$
T_{c_{i}}\left(q_{i}, \dot{q}_{i}\right)=l \sin \left(q_{i}\right) F_{i}\left(q_{i}\right) c_{i}\left(\dot{q}_{i}\right), i=1,2,
$$

where $l=1 \mathrm{~m}$ is the length of leg.

The normal force from the ground through the spring contact is denoted by $F_{i}$ with the following definition

$$
\begin{array}{r}
h(x)=l-l(\cos (x)) \\
\psi(x)=-1000 \min (h(x)-0.02 l, 0) \\
F_{1}(x)=F_{2}(x)=\psi(x) .
\end{array}
$$

The springs are set to have a maximum compression of $0.02 l=2 \mathrm{~cm}$ and it reaches the maximum compression when the leg angle is zero. The function $h(x)$ expresses the height of foot as a function of leg angle. The function $\psi(x)$ utilizes the function $h(x)$ to determine if the spring is compressed, and based on the amount of compression the force is applied.

Figure 2 shows the bump function $\psi(x)$ that represents how the normal contact force varies with the leg angle. The plot shows the maximum force at an angle of zero, as that is the position where the spring compression is maximum, hence the force is maximum. The force eventually decreases to zero, which should be the case when the spring is not compressed at all i.e. the leg is in the swing motion.

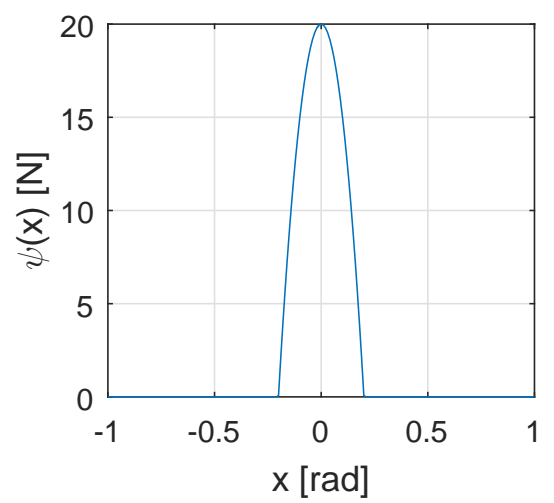

Fig. 2. Normal contact force with respect to leg angle.

The function $c_{i}\left(q_{i}\right)$ determines whether the force from the ground applies to the leg, specifically, with the following definition

$$
c_{i}\left(\dot{q}_{i}\right)=\max \left\{-\operatorname{sign}\left(\dot{q}_{i}\right), 0\right\}, i=1,2 .
$$

In other words, a positive angular velocity suggests that the leg is in forward swing motion and the force from the ground should not be applied to the leg; whereas a negative angular velocity suggests that the leg is in backward swing motion and subjected to the contact force from the ground. This definition in unique direction is critical for generating a forward locomotion. Such ground clearance mechanism for the forward swing motion can be realized, for example, by adding knee joints.

\section{RESULTS}

The results on natural oscillations were achieved through numerical simulations with the following parameters: mass of leg $m=5 \mathrm{~kg}, K_{o}=50\left[\begin{array}{cc}1 & -1 \\ -1 & 1\end{array}\right] \mathrm{Nm} / \mathrm{rad}$, and $G=$ $4.9 m l\left[\begin{array}{ll}1 & 0 \\ 0 & 1\end{array}\right] \mathrm{Nm} / \mathrm{rad}$.

The first result obtained are shown in Figure 3. With suitable damping compensation, the system behaved just like a marginally stable linear locomotor, producing sustained oscillations. These sustained oscillations were able to produce a forward locomotion which is depicted in Figure 4. It shows that the biped robot moves with a forward velocity of $1.2 \mathrm{~m} / \mathrm{s}$.

Next, stronger force from the ground was considered in the second simulation with the coefficient 1,000 in (7) increased to 10,000 . The response of the system is now shown in Figure 5. On close inspection, one can see that the oscillations are distorted. This is because for the same spring compression, greater force is applied in the short time. Even with the distorted oscillations, the pattern was seen to be sustained.

When the maximum compression of the springs was increased to $0.08 \mathrm{l}=8 \mathrm{~cm}$, the response obtained then is shown in Figure 6. The angle of legs remains positive all the time. This is because with a larger spring length, the humanoid is subjected to the normal force for a longer duration. The torque because of the contact force dominates the oscillations in this case, eventually causing the oscillations to die down. This undesired response shows that the spring length should not be too large. With a shorter spring, the impact is captured more accurately, as compared to a longer spring, which even affects the leg length.

\section{CONCLUSION}

A novel model of biped robot was presented in this paper whereby a nonlinear bump function was used to model the discontinuity at the impact. The system showed similarities with the snake-like locomotor in its entrainment to persistent oscillations. However, there were stark differences as well, because of the presence of a nonlinearity. The system still lacks a rigorous stability analysis, and this is the subject of ongoing research. Once that analysis is done, a nonlinear feedback controller can be designed to ensure entrainment to natural oscillations in all conditions. The system complexity 


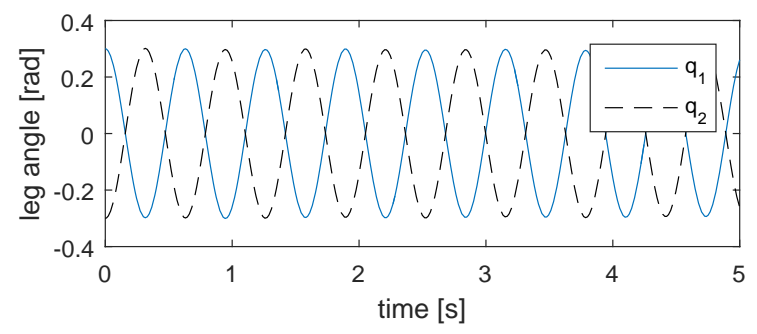

Fig. 3. Natural oscillations as seen in both legs.

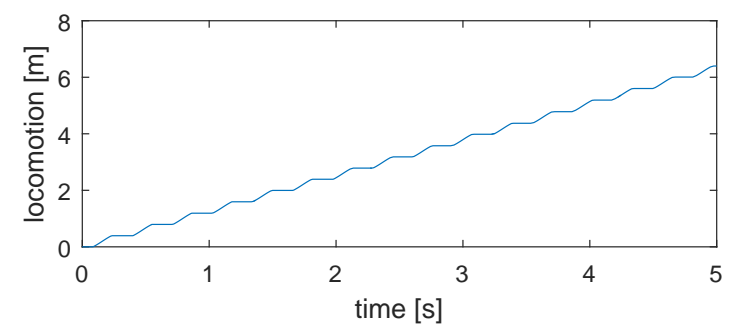

Fig. 4. Locomotion response showing a forward motion.

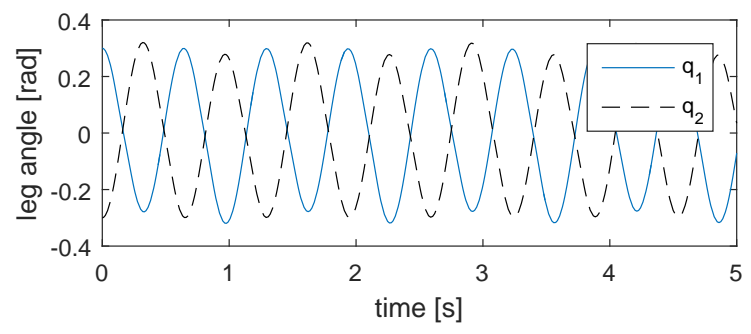

Fig. 5. System response with increased spring stiffness.

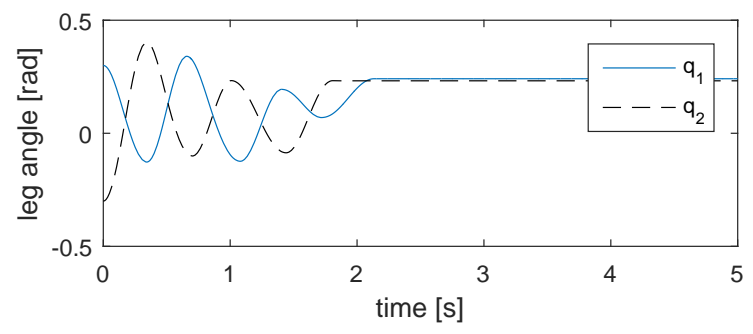

Fig. 6. System response with increased spring length.

can also be increased by adding more joints in the existing structure. Similarly, locomotion analysis is another way forward whereby we can see how oscillation of these links in a specific pattern will give us the desired motion in a direction.

\section{REFERENCES}

[1] K. Jackson, J. Joseph, and S. Wyard, "A mathematical model of arm swing during human locomotion," Journal of Biomechanics, vol. 11, no. 6, pp. 277 - 289, 1978. [Online]. Available: http://www.sciencedirect.com/science/article/pii/0021929078900611

[2] B. Gutnik, H. Mackie, G. Hudson, and C. Standen, "How close to a pendulum is human upper limb movement during walking?" $\{\mathrm{HOMO}\}$ - Journal of Comparative Human Biology, vol. 56, no. 1, pp. 35 - 49, 2005. [Online]. Available: http://www.sciencedirect.com/science/article/pii/S0018442X04000514
[3] S. Mochon and T. A. McMahon, "Ballistic walking: an improved model," Mathematical Biosciences, vol. 52 , no. 3-4, pp. 241-260, 1980. [Online]. Available: http://www.sciencedirect.com/science/article/pii/002555648090070X

[4] T. Anstensrud, "2-d passive compass biped walker : Analysis and robustness of stable gait," Master's thesis, Norwegian University of Science and Technology, Department of Engineering Cybernetics, 2013.

[5] A. Bagheri, A. Hajiloo, and S. Basiri, "Determination of kinematic parameters of a passive bipedal walking robot moving on a declined surface by image processing," WSEAS Transaction on Computer, Vol4, Nov2005, pp1718-1724, 2005.

[6] T. McGeer, "Passive dynamic walking," The International Journal of Robotics Research, vol. 9, no. 2, pp. 62-82, 1990. [Online]. Available: http://ijr.sagepub.com/content/9/2/62.abstract

[7] A. Goswami, B. Espiau, and A. Keramane, "Limit cycles in a passive compass gait biped and passivity-mimicking control laws," Autonomous Robots, vol. 4, no. 3, pp. 273-286, 1997.

[8] A. Goswami, B. Thuilot, and B. Espiau, "A study of the passive gait of a compass-like biped robot: Symmetry and chaos," The International Journal of Robotics Research, vol. 17, no. 12, pp. 1282-1301, 1998. [Online]. Available: http://ijr.sagepub.com/content/17/12/1282.abstract

[9] Y. Hu, G. Yan, and Z. Lin, "Stable running of a planar underactuated biped robot," Robotica, vol. 29, pp. 657-665, 92011.

[10] C. Tang, G. Yan, and Z. Lin, "Walking control for compass-like biped robot with underactuated ankle," pp. 3852-3857, July 2012.

[11] Z. J. Jackowski, "Design, construction, and experiments with a compass gait walking robot," $\mathrm{Ph}$.D. dissertation, Massachusetts Institute of Technology, 2011.

[12] D. Koop and C. Q. Wu, "Passive dynamic biped walking part i: Development and validation of an advanced model," Journal of Computational and Nonlinear Dynamics, vol. 8, no. 4, p. 041007, 2013.

[13] G. A. Cavagna, N. C. Heglund, and C. R. Taylor, "Mechanical work in terrestrial locomotion: two basic mechanisms for minimizing energy expenditure," American Journal of Physiology - Regulatory, Integrative and Comparative Physiology, vol. 233, no. 5, pp. R243-R261, 1977. [Online]. Available: http://ajpregu.physiology.org/content/233/5/R243

[14] Z. Chen and T. Iwasaki, "Robust entrainment to natural oscillations of asymmetric systems arising from animal locomotion," in Decision and Control, 2009 held jointly with the 2009 28th Chinese Control Conference. CDC/CCC 2009. Proceedings of the 48th IEEE Conference on, Dec 2009, pp. 2954-2959.

[15] L. Zhu, Z. Chen, and T. Iwasaki, "Oscillation, orientation, and locomotion of underactuated multilink mechanical systems," IEEE Transactions on Control Systems Technology, vol. 21, no. 5, pp. 15371548, Sept 2013.

[16] M. N. Islam and Z. Chen, "Natural oscillation control of prototype mechanical rectifiers," IEEE Transactions on Control Systems Technology, vol. 20, no. 6, pp. 1559-1566, Nov 2012.

[17] L. B. Freidovich, U. Mettin, A. S. Shiriaev, and M. W. Spong, "A passive 2-dof walker: Hunting for gaits using virtual holonomic constraints," IEEE Transactions on robotics, vol. 25, no. 5, pp. 1202 1208, 2009. 